\title{
Effects of combined physical and cognitive training on fitness and neuropsychological outcomes in healthy older adults
}

This article was published in the following Dove Press journal:

Clinical Interventions in Aging

19 September 2016

Number of times this article has been viewed

\author{
Laurence Desjardins- \\ Crépeau ${ }^{1,2}$ \\ Nicolas Berryman ${ }^{2,3}$ \\ Sarah A Fraser ${ }^{4}$ \\ Thien Tuong Minh Vu $\mathrm{u}^{5,6}$ \\ Marie-Jeanne Kergoat ${ }^{2,6}$ \\ Karen $\mathrm{ZH} \mathrm{Li}^{7}$ \\ Laurent Bosquet ${ }^{8}$ \\ Louis Bherer ${ }^{2,7}$
}

'Department of Psychology, University of Quebec at Montreal, Montreal, QC, Canada; ${ }^{2}$ Research Center, Institut universitaire de gériatrie de Montréal, Montreal, QC, Canada; ${ }^{3}$ Department of Sports Studies, Bishop's University, Sherbrooke, QC, Canada; ${ }^{4}$ Interdisciplinary School of Health Sciences, University of Ottawa, Ottawa, ON, Canada; ${ }^{5}$ Research Center, Centre hospitalier de l'Université de Montréal, Montreal, QC, Canada; ${ }^{6}$ Faculty of Medicine, Université de Montréal, Montreal, QC, Canada; ${ }^{7}$ Department of Psychology and PERFORM Centre, Concordia University, Montréal, QC, Canada; ${ }^{8}$ Faculté des sciences du sport, Université de Poitiers, Poitiers, France

Correspondence: Laurence DesjardinsCrépeau

Centre de recherche de l'Institut universitaire de gériatrie de Montréal, 4565, chemin Queen-Mary, Montréal, QC H3W IW5, Canada

$\mathrm{Tel}+$ I 5 I4 3403540 ext 4 I 48

$\mathrm{Fax}+\mathrm{I} 5$ I4 3403530

Email desjardins.laurence@gmail.com
Purpose: Physical exercise and cognitive training have been shown to enhance cognition among older adults. However, few studies have looked at the potential synergetic effects of combining physical and cognitive training in a single study. Prior trials on combined training have led to interesting yet equivocal results. The aim of this study was to examine the effects of combined physical and cognitive interventions on physical fitness and neuropsychological performance in healthy older adults.

Methods: Seventy-six participants were randomly assigned to one of four training combinations using a $2 \times 2$ factorial design. The physical intervention was a mixed aerobic and resistance training program, and the cognitive intervention was a dual-task (DT) training program. Stretching and toning exercises and computer lessons were used as active control conditions. Physical and cognitive measures were collected pre- and postintervention.

Results: All groups showed equivalent improvements in measures of functional mobility. The aerobic-strength condition led to larger effect size in lower body strength, independently of cognitive training. All groups showed improved speed of processing and inhibition abilities, but only participants who took part in the DT training, independently of physical training, showed increased task-switching abilities. The level of functional mobility after intervention was significantly associated with task-switching abilities.

Conclusion: Combined training did not yield synergetic effects. However, DT training did lead to transfer effects on executive performance in neuropsychological tests. Both aerobic-resistance training and stretching-toning exercises can improve functional mobility in older adults.

Keywords: aging, combined intervention, physical performance, cognitive performance, dual task, executive functions

\section{Introduction}

Aging is often accompanied by physiological changes that can lead to decline in physical and cognitive capacities, often resulting in loss of autonomy or institutionalization. In the last 20 years, a large body of research has been oriented toward finding effective ways to enhance cognitive functioning in older adults or to prevent cognitive decline. Among them, physical exercise and cognitive training have demonstrated important benefits for cognitive functioning in the older adult population. ${ }^{1-3}$ However, only recently have researchers investigated the potential synergetic effect of combining more than one approach for preventing cognitive decline and enhancing cognitive abilities. The present study aims at examining the effects of the combination of a physical training regimen and a cognitive training intervention on cognitive performance in healthy older individuals. 
Many cross-sectional ${ }^{4-6}$ as well as longitudinal studies ${ }^{7}$ have reported that physical activity is associated with better cognitive functions. A meta-analysis of prospective studies recently reported that regular practice of physical activity was associated with a $35 \%-38 \%$ reduction in the risk of cognitive decline and dementia. ${ }^{8}$ Results from intervention studies provide additional support to the notion that physical exercise can lead to cognitive improvement in older adults. Significant improvements in cognition after aerobic exercise have been reported, ${ }^{9}$ and it seems that some executive functions appear to benefit more from physical training than other cognitive functions. ${ }^{10-13}$ Brain imaging studies have reported an association between exercise intervention or regular practice of exercise and repletion of brain volume loss and increased activation in frontal and parietal cortices. ${ }^{14,15}$ Increase in cardiovascular fitness has largely been identified as a fundamental mechanism for the cognitive enhancement, ${ }^{16}$ but increasing evidence suggests that strength training also exerts beneficial physiological transformations that can boost cognition. ${ }^{17-19}$ Thus, the most recent approaches in designing physical intervention studies lean toward mixed programs that combine aerobic and resistance training regimens.

Several studies have reported increased cognitive performance after cognitive training in older adults, ${ }^{20-22}$ although the extent to which the benefits transfer to nontrained tasks is still a matter of debate. ${ }^{23,24}$ Nevertheless, it seems safe to suggest that computerized cognitive training represents an effective way to improve specific cognitive functions or mechanisms. In its more general format, computerized cognitive training uses structured training programs with appropriate feedback to induce improvement in a specific task. It is generally assumed that observing improvement in a transfer task, different than the one used for training, suggests skill learning or improved cognitive functions or process, beyond the learning of a simple stimulus-response association. Many studies have reported that cognitive training can improve a variety of cognitive abilities or processes, such as memory, ${ }^{25-27}$ working memory, ${ }^{28,29}$ attention and speed of processing, ${ }^{30,31}$ and executive control. ${ }^{23,32,33}$

Accumulating evidence thus suggests that physical exercise interventions and cognitive training can boost cognitive function in older adults. However, a limited number of studies have looked at the additional or interactive effects of both types of training in a single trial, and many of them were conducted with a cognitively impaired population. ${ }^{34-36}$ From a preventive perspective, it seems important to examine the effects of combined interventions on the cognitive performance of healthy older adults. Combined training intervention studies that were conducted with healthy older participants have led to mixed results so far. Some studies showed evidence for significant benefits of the combined condition over the single-domain training ${ }^{37}$ and even sustained gains in cognitive performance for the combined training group 5 years after the termination of the intervention. ${ }^{38}$ On the other hand, other studies reported no additional cognitive enhancement after combined training when compared to cognitive training alone..$^{39,40}$

Taken together, results from intervention studies combining both physical and cognitive training remain equivocal in terms of potential added value of doing both physical and cognitive training rather than each one alone. The heterogeneity of study designs and type of physical and cognitive training likely explain these mixed results. One important factor that could greatly contribute to heterogeneity is that most cognitive interventions were multicomponent by nature, targeting several cognitive domains or functions, some of which are less sensitive to physical activity intervention (eg, memory) than others (eg, executive control). Literature has shown that both physical exercise and cognitive training interventions seem to be particularly effective in improving executive functions. ${ }^{10,28,41,42}$ Executive functions are frontally mediated functions involved in complex goaloriented behavior that encompasses different mechanisms, such as inhibiting an automatic response, alternating between multiple tasks, updating information in working memory, and coordinating several tasks. ${ }^{43}$ It has been reported that executive functioning can be altered early in the course of aging, and this has often been recognized as a significant predictor of preserved instrumental activities of daily living, such as preparing meals, managing finances, doing housework, and taking medications. ${ }^{44} 48$ These functions thus play an essential role in independent functioning in older adults. Among executive functions, the ability to coordinate several tasks, which implies to divide the attention on multiple tasks, has received a great amount of interest in the past decades, especially in older adults. Attention-sharing abilities decline with age $e^{49,50}$ but are still required in a variety of everyday tasks. In fact, divided attention is involved in complex motor tasks, such as walking, and deficits in the ability to coordinate several tasks are associated with increasing risk of falls. ${ }^{51,52}$ Improving executive control of attention would therefore be a promising way to foster autonomy and wellbeing among aging individuals and should thus be a primary focus in intervention studies. However, trials conducted so far have not put forward executive functions as the main 
target for cognitive and physical training. Moreover, the training regimens were not always based on validated and published approaches. In the present study, a dual-task (DT) training was chosen as the cognitive training because of its effectiveness in improving executive control of attention. In fact, studies involving a DT paradigm have demonstrated a training-induced improvement in attention-sharing abilities in older adults with transfer in similar untrained tasks. ${ }^{32,33,53-55}$ Furthermore, while many studies have reported near transfer (to a task with similar response requirements), a recent study accounted for a more distal transfer after five sessions of DT training, with transfer effects extending to an untrained task that involved different stimuli and response modalities. ${ }^{56}$

In addition, the implementation of active control conditions is of major importance in intervention studies to ensure that observed effects are driven by the active ingredient of the training rather than any other feature of the intervention. Some studies conducted thus far have compared intervention groups with passive control groups that remain on a waiting list and do not interact with the other participants or the experimenters during the intervention period. ${ }^{40}$ This leads to increased social interactions for the training groups compared to controls. ${ }^{57}$ Controlling exposure to social interaction is paramount especially if the combined interventions lead to greater social interaction (up to twice as much in some studies) than each condition alone, as was the case in previous reports. ${ }^{37,39}$ These questions have already been raised by Rahe et al, ${ }^{58}$ but their nonrandomized combined intervention with two small groups (cognitive training and combined cognitive and physical training) did not address adequately these limitations.

In the present study, two active control conditions were designed for both the physical and the cognitive training interventions. We used a $2 \times 2$ factorial design to create four possible combinations of conditions. By doing so, we ensured that every participant received the same amount of social interaction and exposure to laboratory environment and staff in each condition of the study. In addition, the cognitive intervention targeted only one domain (executive control) and function (DT) and was based on an extensive literature supporting its efficacy to improve attentional control..$^{32,33,53-55}$ The mixed aerobic and strengthening physical training was also designed based on previous studies, suggesting its enhancing effects on cognition. ${ }^{59}$ The goal of the study was to examine the potential synergetic effect of a combined physical and cognitive intervention on fitness and neuropsychological outcomes in a sample of healthy sedentary older adults. It was hypothesized that combined physical exercise and cognitive training targeting DT processing would lead to better executive functioning than each intervention alone.

\section{Methods \\ Participants}

Participants were recruited from public advertisements (flyers and newspapers) and from the research center's participant pool. A telephone-based screening interview was used to assess the eligibility of each candidate. Exclusion criteria were as follows: history of neurological disease or major surgery in the year preceding the study, auditory or visual impairments that were not corrected, smoking, severe mobility limitations, and any other contraindication to perform physical activity, being currently engaged in any type of structured physical activity. A total of 136 communitydwelling individuals, 60 years and older, were enrolled in this trial. Eleven participants withdrew prior to the first pretest assessment, leaving 125 participants in the sample.

Among the 125 participants who were enrolled in the study, 91 participants completed the program. Among those, three participants failed to participate in the posttest evaluations and two participants had invalid data due to illness at posttest examinations and were thus excluded from analyses. Finally, ten participants were excluded due to high score on the geriatric depression scale ${ }^{60}$ (score $>11$, a score suggestive of mild or moderate depression), leaving 76 participants in the final sample. Table 1 summarizes participants' baseline characteristics. The study was approved by the ethical review board of the Research Center of the Institut universitaire de gériatrie de Montréal and all participants provided written informed consent.

\section{Medical evaluation and cognitive screening measures}

Participants underwent an exhaustive medical assessment conducted by a geriatrician. Multiple medical conditions related to cardiovascular, pulmonary, musculoskeletal and gastrointestinal disorders were identified as present or absent. For the scope of the present study, only cardiovascular risk factors, known to influence the integrity of brain structures and functions, are presented. Based on a previous study, ${ }^{61}$ a cardiovascular risk score that represents the sum of the following conditions was computed: hypertension, dyslipidemia, diabetes, angina, heart failure, arrhythmia, myocardial infarction, valvular disease, stroke/transient ischemic attack, abnormal body mass index ( $\geq 30 \mathrm{~kg} / \mathrm{m}^{2}$ ), and abnormal waist circumference $(>102 \mathrm{~cm}$ for men and $>88 \mathrm{~cm}$ for women). Cognitive screening included a measure of global cognition (mini-mental state evaluation), ${ }^{62}$ a measure of verbal concept formation (similarities subtest 
Table I Baseline characteristics of participants

\begin{tabular}{|c|c|c|c|c|c|c|}
\hline \multirow[t]{2}{*}{ Characteristics } & \multicolumn{2}{|c|}{ AR training $(n=38)$} & \multicolumn{2}{|c|}{ ST exercises $(n=38)$} & \multicolumn{2}{|l|}{$P$-value } \\
\hline & $\begin{array}{l}\text { DT training } \\
(n=22)\end{array}$ & $\begin{array}{l}C L \\
(n=16)\end{array}$ & $\begin{array}{l}\text { DT training } \\
(n=20)\end{array}$ & $\begin{array}{l}C L \\
(n=18)\end{array}$ & $\begin{array}{l}\text { AR training vs } \\
\text { ST exercises }\end{array}$ & $\begin{array}{l}\text { DT training } \\
\text { vs } C L\end{array}$ \\
\hline Age (years) & $72.7(7.4)$ & $70.9(7.4)$ & $73.2(6.3)$ & $72.5(7.0)$ & 0.521 & 0.452 \\
\hline Sex (Female/Male) & $13 / 9$ & $8 / 8$ & $17 / 3$ & $15 / 3$ & $\mathrm{n} / \mathrm{a}$ & $\mathrm{n} / \mathrm{a}$ \\
\hline Education (years) & | 4.4 (2.8) & $15.9(2.1)$ & |4.| (4.3) & |4.| (3.8) & 0.178 & 0.367 \\
\hline CVR (score) & $2.1(1.7)$ & $1.7(1.2)$ & $2.5(1.5)$ & $2.3(1.3)$ & 0.153 & 0.365 \\
\hline GDS (score) & $4.6(3.3)$ & $4.4(2.6)$ & $3.4(3.2)$ & $2.7(2.9)$ & $0.048^{*}$ & 0.574 \\
\hline \multicolumn{7}{|l|}{ Cognitive screening measures } \\
\hline MMSE (score) & $28.8(1.3)$ & $28.8(1.0)$ & $29.4(0.7)$ & $28.5(1.4)$ & 0.616 & 0.128 \\
\hline Similarities (score) & $23.4(4.5)$ & $22.2(4.8)$ & $22.0(4.7)$ & $22.3(5.7)$ & 0.548 & 0.694 \\
\hline Digit span-forward (score) & $9.3(1.8)$ & $9.5(1.9)$ & $9.3(2.4)$ & $9.0(2.5)$ & 0.608 & 0.907 \\
\hline Digit span-backward (score) & $6.1(1.6)$ & $6.6(1.8)$ & $7.0(2.3)$ & $5.9(1.6)$ & 0.773 & 0.461 \\
\hline DSST (score) & $58.5(12.1)$ & $60.3(18.7)$ & $54.9(13.1)$ & $57.0(14.9)$ & 0.307 & 0.563 \\
\hline
\end{tabular}

Notes: Data presented as mean (standard deviation) unless stated otherwise. $* P<0.05$.

Abbreviations: AR, aerobic and resistance; CVR, cardiovascular risk; CL, computer lessons; DSST, digit symbol substitution test; DT, dual-task; GDS, geriatric depression scale; MMSE, mini-mental state evaluation; n/a, not available; ST, stretching and toning.

of the Wechsler Adults Intelligence Scale III [WAIS-III]), ${ }^{63}$ a measure of processing speed (Digit Coding subtest of the WAIS-III), and a measure of short-term and working memory (Digit Span subtest of the WAIS-III).

\section{Protocol}

All participants completed one of the four combinations of physical exercise and cognitive training programs. The physical exercise component was either a mixed aerobic and resistance (AR) training program or its control condition composed of stretching and toning (ST) exercises. The cognitive component was either a DT training program (DT) or computer lessons (CL) as a control condition. The study was carried on in waves of 16-32 participants randomly assigned to one of the four training combinations using the website randomization.com. The training took place in the subgroups of 4-8 participants at a time.

\section{Intervention}

The intervention consisted of three sessions per week for a duration of 12 weeks, which included two 60-minute sessions of physical exercise interspersed by one 60 -minute cognitive stimulation session, for a total of 24 physical training sessions and twelve cognitive training sessions. Participants were considered as having completed the training program if they attended to a minimum of $75 \%$ of the training sessions in each type of training, that is, a minimum of 18 physical exercise sessions and nine cognitive training sessions.

\section{Physical training}

The physical exercise training programs took place in a gymnasium located in a geriatric hospital institution and were supervised by a certified kinesiologist.

\section{Mixed AR training}

The AR training program was primarily intended to increase the participants' cardiorespiratory fitness and lower body muscle strength. Each 1-hour session started with a 5-minute warm-up, followed by a 15 -minute resistance training using resistance cables and targeting multiple lower body muscle groups (quadriceps, hamstrings, hip extensors and flexors, ankle plantar flexors, etc). Then participants were taking part to the 30-minute cardiovascular training portion on a treadmill, during which they were asked to walk and maintain a moderate-to-high intensity of effort on the Borg scale of perceived exertion. ${ }^{64}$ The intensity of exercise gradually increased over the sessions by augmenting the slope of the treadmill and the resistance of the cables. The kinesiologist constantly monitored the level of effort and tiredness of participants in order to adjust exercise intensity accordingly. The session ended with a 5-minute cool down period.

\section{ST exercises}

This program involved a series of exercises aiming at improving flexibility and general motor skills without a specific focus on increasing cardiorespiratory capacity or strength. After a 5-minute warm-up on the machine of their choice, participants performed stretching exercises, based on Stanziano et al, ${ }^{65}$ that targeted the entire body for 50 minutes. A large part of the exercises were performed in a seated position. The session ended with a 5-minute relaxation session on a yoga mat.

\section{Cognitive training \\ DT training}

Each weekly session of DT training took place in a room with ten computer stations and was supervised by a student 
in neuropsychology. Participants were trained on a computerized visual DT analog to the one used in several previous studies. $^{32,54}$ The task consisted of two visual discrimination tasks performed separately and concurrently. The first task was a number discrimination task $(3,5$, and 8$)$, and the second was a shape discrimination task (circle, square, and diamond). Participants had to respond to the number or shapes presented on the screen with a key press on the appropriate button identified on the keyboard. The DT was made of four different blocks, each containing different types of trials. Each task was performed alone in the two first blocks, named single pure (SP) blocks (numbers and then shapes). In the single mixed (SM) blocks, participants still had to perform one task at a time, but they were unaware of the nature of the stimuli that would appear on the screen (number OR shape) at each trial. Finally, in the dual mixed (DM) blocks, the two tasks had to be performed at the same time at each trial. Participants were instructed to avoid prioritizing one task over the other and to provide an answer using the keyboard as quickly and accurately as possible. Each training session involved 72 SP trials, 240 SM trials, and 864 DM trials. The training task provided continuous feedback of performances during the DM blocks. Feedback was provided with a histogram that changed color (green, yellow, and red) to inform participants on their response speed. The goal was to maintain the bar of the histogram in the green zone and to avoid it becoming red. The color of the histogram is determined by the average reaction time of the last three trials of the DM block that is compared to the median reaction time of the SM block. Thus, when the average reaction time of the three previous trials is closer to the median reaction time of the SM block, the histogram became green. In addition to this continuous feedback on speed, a histogram informing on the progression with regard to speed and accuracy was presented on the screen at the end of each session. Such adapted and continuous feedback represents a hallmark of effective cognitive training because it favors the acquisition of a skill rather than promoting a specific stimulus-response mapping through extensive practice.

\section{$C L$}

The CL condition consisted of introductory exercises to computers and diverse software (eg, Word and Excel), as well as an initiation to Internet (search engines, web sites, online games, etc). These sessions took place in the same computer room as the DT training program and were taught by a student in neuropsychology.

\section{Outcome measures}

Participants took part in three pretest evaluation sessions within a 2-week interval before the beginning of the intervention. Medical and screening cognitive measures were collected in the first session, neuropsychological assessment was completed during the second session, and physical functioning tests took place in the third session. Two posttest evaluation sessions (neuropsychological and physical assessments) were completed within 2 weeks following the training programs. The evaluators at both pretest and posttest were blind to the group membership of participants.

\section{Neuropsychological assessment}

Neuropsychological assessment targeted memory, processing speed, and executive functions (see Lezak et $\mathrm{al}^{66}$ for an exhaustive description of each test): 1) The Rey AuditoryVerbal Learning Test assesses verbal learning and retrieval. A list of 15 words is read by the examiner, and the participants are asked to repeat the maximum number of words afterward. There are five successive trials. A second 15-word list is then presented for one trial, in order to create interference. After this single trial, participants are asked to retrieve a maximal number of words from the first list. To evaluate long-term retention, participants have to recall the words from the first list after a 30-minute delay. Measures selected from this test are the total number of words retrieved over the five trials, the number of words recalled after interference, and the number of words recalled after delay. 2) The Color-Word Interference Test (CWIT) of the Delis-Kaplan Executive Functions System ${ }^{67}$ is based on the Stroop procedure. ${ }^{68}$ The test includes the following four conditions: a) color naming of colored rectangles, b) reading of color words printed in black ink, c) inhibition of reading for naming incongruent ink colors in which color words are printed in, and d) switching between reading and color naming of color words printed in an incongruent ink color. This test assesses the speed of processing for the former two conditions and executive functioning (inhibition and switching or cognitive flexibility) for the latter two conditions. Time to complete each condition and number of errors were recorded. 3) The trail-making test (TMT $)^{69}$ is composed of two parts. In part A, participants are asked to link numbers from 1 to 25 in serial order as quickly as possible. This portion assesses the speed of processing. Part B assesses task-switching abilities. Participants have to alternatively link numbers and letters (1-A-2-B-3-C, etc) as fast as they can. Time to complete each part and number of errors are recorded. 4) The Baddeley $\mathrm{DT}^{70}$ was used to assess the divided attention abilities and is composed of the 
Table 2 Mean and SD of physical performance measures

\begin{tabular}{|c|c|c|c|c|c|c|c|c|}
\hline \multirow[t]{3}{*}{ Measures } & \multicolumn{4}{|c|}{ AR training $(n=38)$} & \multicolumn{4}{|c|}{ ST exercises $(n=38)$} \\
\hline & \multicolumn{2}{|c|}{ DT training $(n=22)$} & \multicolumn{2}{|l|}{$C L(n=16)$} & \multicolumn{2}{|c|}{ DT training $(n=20)$} & \multicolumn{2}{|l|}{$C L(n=18)$} \\
\hline & Pre & Post & Pre & Post & Pre & Post & Pre & Post \\
\hline Handgrip strength $(\mathrm{kg})^{\mathrm{a}, * * * *}$ & $42.3(16.4)$ & $41.7(19.3)$ & $49.9(15.8)$ & $50.2(16.0)$ & $34.6(10.5)$ & $33.9(9.9)$ & $37.4(12.8)$ & $36.1(10.3)$ \\
\hline TUG (seconds) & $6.9(1.2)$ & $6.2(0.9)$ & $6.6(1.7)$ & $6.2(0.9)$ & $7.5(1.7)$ & $7.0(1.5)$ & $6.7(1.1)$ & $6.1(0.8)$ \\
\hline 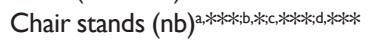 & $13.9(3.3)$ & $20.2(5.2)$ & $13.0(2.9)$ & $18.9(7.3)$ & $11.0(3.4)$ & $12.4(3.5)$ & $13.8(3.9)$ & $16.3(5.2)$ \\
\hline 6-MWT $(m)^{c, * *}$ & $501.2(68.7)$ & $535.4(71.7)$ & $532.3(74.3)$ & $566.4(91.8)$ & $499.1(85.9)$ & $515.0(77.8)$ & $502.2(69.7)$ & $533.5(64.3)$ \\
\hline PPT (score) $)^{c, * *}$ & $32.4(2.4)$ & $34.2(1.9)$ & $31.9(3.5)$ & $33.6(1.8)$ & $31.5(2.1)$ & $32.8(2.1)$ & $32.4(2.8)$ & $33.3(3.2)$ \\
\hline
\end{tabular}

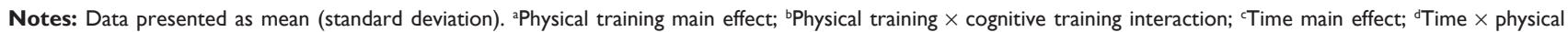
training interaction: $* P<0.05 ; * * P<0.01$; $* * * P<0.00$ I.

Abbreviations: AR, aerobic and resistance; CL, computer lessons; DT, dual-task; 6-MWT, 6-minute walk test; PPT, physical performance test; ST, stretching and toning; TUG, timed up and go.

following two tasks performed alone, and then together: a) a cancelation task in which a maximum of $\mathrm{Xs}$ are traced along a path in 120 seconds and b) a digit span task in which participants have to repeat series of digits during 120 seconds. A DT index is then computed using a specific equation suggested in the scoring manual. A lower score on this index indicates greater DT interference, that is, poorly divided attention performance. The neuropsychological assessment lasted $\sim 1$ hour.

\section{Physical and functional assessment}

Physical and functioning tests included the 6-minute walk test (6-MWT), ${ }^{71}$ the modified physical performance test (PPT), ${ }^{72}$ the timed up and go (TUG),${ }^{73}$ handgrip strength, and chair stand. The 6-MWT is a self-paced walking test in which participants must travel the longest distance possible in 6-minute time. The PPT includes nine tasks rated from 0 to 4 points, for a maximum score of 36 . The following seven tasks were timed: $15 \mathrm{~m}$ walk, put on and remove a coat, pick up a coin on the floor, stand up from a chair (five times), pick up a heavy book from a shelf, climb nine stairs and standing balance with feet side-by-side, and semitandem and tandem positions. The two nontimed tasks are as follows: performing a $360^{\circ}$ turn and climbing up and down four flights of stairs. In the TUG, participants had to stand up from a chair and walk $3 \mathrm{~m}$ in a straight line, then around a cone, and $3 \mathrm{~m}$ back to sit down on the chair, as quickly as possible. Handgrip strength was assessed with a hand-held dynamometer (Smedley-type hand dynamometer; ERP, Laval, QC, Canada). The best of three trials with the dominant hand was recorded. In the chair stand test, maximum chair stands in 30 seconds was performed and used as a proxy of lower extremity strength. The total duration of the physical assessment was $\sim 45$ minutes.

\section{Data analyses}

Repeated-measures analyses of variance $(2 \times 2 \times 2)$, with physical training (AR vs ST) and cognitive training (DT vs CL) as between-subject factors and time (pretest and posttest) as a within-subject factor, were performed on physical and neuropsychological outcomes. We looked at the main effect of time to assess change from pretest to posttest for each measure. A physical training $\times$ time and/or a cognitive training $\times$ time interaction would suggest a beneficial effect of one or both types of training on physical and/or cognitive performance. A physical training $\times$ cognitive training $\times$ time interaction would be considered as a demonstration of a synergetic effect of physical training and cognitive training on physical and/or cognitive performances. Pretest and posttest raw scores on physical functioning tests are presented in Table 2 , and scores on neuropsychological measures are presented in Table 3. The alphas of 0.05 are considered significant, and all follow-up analyses were Bonferroni corrected.

\section{Results}

Given that there was a significant difference in geriatric depression scale score between physical training groups (Table 1), this measure was added as a covariate in all analyses. The physical training $\times$ cognitive training interaction was not significant for any of the variables at baseline, hence $P$-values of the interaction are not reported in Table 1 . In the following sections, only significant results are summarized, with follow-up analyses for significant interactions.

\section{Intervention effects on physical performance}

There was a main effect of time on the 6-MWT, $F(1,71)=10.70$, $P=0.002, \eta_{\mathrm{p}}^{2}=0.131$, and on the PPT, $F(1,71)=11.55$, $P=0.001, \eta_{\mathrm{p}}^{2}=0.140$, and no interaction effect, suggesting equivalent improvement in all groups. Handgrip strength and TUG did not significantly improve after intervention. A physical training $\times$ time interaction was revealed for the chair stand test, $F(1,71)=16.80, P<0.001, \eta_{\mathrm{p}}^{2}=0.191$, due to a greater improvement after intervention in the AR group, $F(1,37)=58.83, P<0.001, \eta_{\mathrm{p}}^{2}=0.614$, than in the ST group, 
Table 3 Mean and SD of neuropsychological measures

\begin{tabular}{|c|c|c|c|c|c|c|c|c|}
\hline \multirow[t]{3}{*}{ Measures } & \multicolumn{4}{|c|}{ AR training $(n=38)$} & \multicolumn{4}{|c|}{ ST exercises $(n=38)$} \\
\hline & \multicolumn{2}{|c|}{ DT training $(n=22)$} & \multicolumn{2}{|l|}{$C L(n=16)$} & \multicolumn{2}{|c|}{ DT training $(n=20)$} & \multicolumn{2}{|l|}{$C L(n=18)$} \\
\hline & Pre & Post & Pre & Post & Pre & Post & Pre & Post \\
\hline \multicolumn{9}{|l|}{ Memory } \\
\hline RAVLT-total 5 trials (nb) & $50.3(9.6)$ & $47.4(8.4)$ & $47.6(10.0)$ & $47.8(9.9)$ & $51.3(10.1)$ & $48.7(9.5)$ & $52.3(10.3)$ & $51.9(8.8)$ \\
\hline RAVLT-immediate recall (nb) & $10.2(3.3)$ & $8.9(3.1)$ & $10.3(2.8)$ & $8.8(3.5)$ & $10.6(2.5)$ & $10.0(3.1)$ & $10.9(3.0)$ & $9.8(3.0)$ \\
\hline RAVLT-delayed recall $(\mathrm{nb})$ & $10.6(3.6)$ & $9.2(2.7)$ & $9.6(3.1)$ & $9.4(3.3)$ & II.2(2.5) & $9.8(2.6)$ & II.8 (2.9) & $10.4(3.3)$ \\
\hline \multicolumn{9}{|l|}{ Processing speed } \\
\hline CWIT-color naming (seconds) & $31.6(6.0)$ & $31.6(6.4)$ & $32.7(7.0)$ & $33.2(7.3)$ & $32.4(6.0)$ & $30.6(4.3)$ & $32.9(8.5)$ & $32.9(11.2)$ \\
\hline CWIT-reading (seconds) & $21.9(3.1)$ & $21.4(2.8)$ & $22.9(4.6)$ & $23.0(4.5)$ & $22.4(3.0)$ & $22.7(3.2)$ & $23.0(3.9)$ & $23.0(4.5)$ \\
\hline TMT-part A (seconds), & $39.2(10.6)$ & $34.8(7.4)$ & $36.4(10.2)$ & $35.3(11.1)$ & $43.8(14.6)$ & $37.1(8.7)$ & $38.7(10.7)$ & $37.1(11.6)$ \\
\hline BDT-single task (Xs) & $161.6(24.1)$ & $168.6(23.2)$ & I52.I (29.1) & I56.7 (27.8) & I55.3 (21.2) & $160.2(18.7)$ & I55.I (29.5) & $160.6(27.4)$ \\
\hline \multicolumn{9}{|l|}{ Executive functions } \\
\hline CWIT-inhibition (seconds)a,* & 6I.I (13.9) & $59.2(12.4)$ & $62.9(15.2)$ & $60.5(11.7)$ & $68.3(22.0)$ & $64.6(22.0)$ & $67.7(22.4)$ & $64.2(15.4)$ \\
\hline CWIT-switching (seconds) & $68.1(28.0)$ & $63.8(13.4)$ & $61.7(13.6)$ & $64.9(16.4)$ & $71.3(24.1)$ & $66.0(22.5)$ & $65.8(11.3)$ & $67.6(16.3)$ \\
\hline TMT-part B (seconds) & $95.0(27.8)$ & $90.8(23.9)$ & $84.6(24.6)$ & $80.4(14.5)$ & $95.8(58.3)$ & $90.1(42.2)$ & $89.0(30.3)$ & $96.6(42.1)$ \\
\hline BDT-DT index & $45.9(10.1)$ & $43.2(19.6)$ & $39.3(16.4)$ & $45.5(10.2)$ & $43.7(15.2)$ & $48.5(9.0)$ & $45.8(9.1)$ & $46.8(8.8)$ \\
\hline
\end{tabular}

Notes: Data presented as mean (standard deviation). ${ }^{\mathrm{T} T i m e}$ main effect; ${ }^{\mathrm{b}} \mathrm{Time} \times$ cognitive training interaction: $* P<0.05$ and $* * P<0.01$.

Abbreviations: AR, aerobic and resistance; BDT, Baddeley DT; CL, computer lessons; CWIT, Color-Word Interference Test; DT, dual-task; nb, number of words; RAVLT, Rey Auditory-Verbal Learning Test; ST, stretching and toning; TMT, trail-making test; Xs, number of Xs.

$F(1,37)=16.70, P<0.001, \eta_{\mathrm{p}}^{2}=0.311$. The physical training $\times$ cognitive training $\times$ time interaction was not significant.

\section{Intervention effects on cognitive performance}

Results show a main effect of time on part A of the TMT, $F(1,70)=7.87, P=0.007, \eta_{\mathrm{p}}^{2}=0.101$, and the inhibition condition of the CWIT, $F(1,71)=6.52, P=0.013, \eta_{\mathrm{p}}^{2}=0.084$, indicating a significant improvement after intervention for all groups. A cognitive training $\times$ time interaction was observed for the switching condition of the CWIT, $F(1,71)=7.21, P=0.009, \eta_{\mathrm{p}}^{2}=0.092$. Simple effects analysis indicates a significant improvement after intervention in the DT group, $F(1,41)=4.85, P=0.033, \eta_{\mathrm{p}}^{2}=0.106$, whereas the CL group did not improve after intervention, $F(1,33)=2.20$, $P=0.148, \eta_{\mathrm{p}}^{2}=0.062$.

\section{Relationship between physical and cognitive performance after intervention}

Multiple hierarchical linear regression analyses were conducted to explore the relationship between physical and cognitive performances after intervention. Results from repeated analysis of variances were used as a guide to select relevant physical measures to include as predictors and cognitive measures to include as outcome variables. Thus, only physical and cognitive measures that showed a significant change after intervention (any significant effect involving time) were selected in these exploratory analyses.
For each regression, age, years of education, and sex were included in a first block of independent variables because these parameters are known to be determinants of cognitive performance. The posttest scores of the PPT, the 6-MWT, and chair stands showed a significant change after intervention and were then included in a second block of predictors to assess the relationship with cognitive performance when age, education, and sex are controlled for. Three cognitive scores showed improvement after intervention, that is, part A of the TMT, as well as inhibition and switching conditions of the CWIT, and were selected as outcome variables. We thus conducted three regression analyses, one for each of these posttest outcome measures.

For each block, the significance of the $F$ ratio is considered to see if each set of predictors explains a significant portion of the variance. The magnitude of the standardized $\beta$-coefficients is also considered to understand the relative contribution of each independent variable in explaining cognitive performance after intervention.

Table 4 presents summary results of the three hierarchical regressions. Neither sociodemographic factors nor posttest physical measures explained a significant proportion of the variance of posttest RT of part A of the TMT and posttest RT in inhibition condition of the CWIT. However, age, sex, and education (Model 1) explained a significant $24 \%$ of the variance of RT in part A of the TMT, $F(3,72)=7.729$, $P<0.001$. More interestingly, the addition of the posttest physical measures explained an additional $11 \%$ of the variance in Model 2, $F(6,69)=6.403, P<0.001$. 
Table 4 Summary of multiple hierarchical regression analyses predicting neuropsychological scores at posttest

\begin{tabular}{|c|c|c|c|c|c|c|}
\hline \multirow[t]{2}{*}{ Factors } & \multicolumn{3}{|l|}{ Model I } & \multicolumn{3}{|l|}{ Model 2} \\
\hline & B & $\beta$ & $P$-value & B & $\beta$ & $P$-value \\
\hline \multicolumn{7}{|l|}{ TMT-A (post) } \\
\hline Age & 0.390 & 0.284 & $0.015^{*}$ & 0.303 & 0.221 & 0.090 \\
\hline Education & 0.076 & 0.027 & 0.813 & 0.264 & 0.094 & 0.428 \\
\hline Sex & -0.529 & -0.026 & 0.824 & -1.032 & -0.050 & 0.672 \\
\hline Chair stands & & & & 0.295 & 0.189 & 0.160 \\
\hline 6-MWT & & & & -0.004 & -0.036 & 0.801 \\
\hline \multirow[t]{4}{*}{ PPT } & & & & -1.206 & -0.292 & 0.052 \\
\hline & $R^{2}=0.082$ & & & $R^{2}=0.148$ & & \\
\hline & $F=2.115$ & & & $\Delta R^{2}=0.066$ & & \\
\hline & & & & $F=1.964$ & & \\
\hline \multicolumn{7}{|c|}{ CWIT-inhibition (post) } \\
\hline Age & 0.604 & 0.264 & $0.022 *$ & 0.409 & 0.178 & 0.168 \\
\hline Education & -0.699 & -0.149 & 0.192 & -0.595 & -0.127 & 0.283 \\
\hline Sex & 3.322 & 0.097 & 0.395 & 5.239 & 0.153 & 0.198 \\
\hline Chair stands & & & & -0.260 & -0.100 & 0.454 \\
\hline 6-MWT & & & & -0.046 & -0.222 & 0.116 \\
\hline \multirow[t]{4}{*}{ PPT } & & & & 0.594 & 0.087 & 0.557 \\
\hline & $R^{2}=0.096$ & & & $R^{2}=0.142$ & & \\
\hline & $F=2.535$ & & & $\Delta R^{2}=0.046$ & & \\
\hline & & & & $F=1.899$ & & \\
\hline \multicolumn{7}{|c|}{ CWIT-switching (post) } \\
\hline Age & 1.153 & 0.466 & $0.000 * * *$ & 0.782 & 0.316 & $0.006 * *$ \\
\hline Education & -0.859 & -0.170 & 0.106 & -0.504 & -0.010 & 0.332 \\
\hline Sex & 3.138 & 0.084 & 0.417 & 5.638 & 0.152 & 0.140 \\
\hline Chair stands & & & & 0.047 & 0.017 & 0.884 \\
\hline 6-MWT & & & & -0.082 & -0.366 & $0.003^{* *}$ \\
\hline \multirow[t]{4}{*}{ PPT } & & & & -0.298 & -0.040 & 0.753 \\
\hline & $R^{2}=0.244$ & & & $R^{2}=0.358$ & & \\
\hline & $F=7.729 * * *$ & & & $\Delta R^{2}=0.114$ & & \\
\hline & & & & $F=6.403 * * *$ & & \\
\hline
\end{tabular}

Notes: $* P<0.05 ; * * P<0.01 ; * * * P<0.001$.

Abbreviations: CWIT, Color-Word Interference Test; 6-MWT, 6-minute walk test; PPT, physical performance test; TMT-A, trail-making test-part A.

Examination of regression coefficients reveals that age is significantly associated with posttest scores of the switching condition of the CWIT, $\beta=0.316, P=0.006$, as well as the distance traveled in the 6-MWT at posttest, $\beta=-0.366$, $P=0.003$.

\section{Discussion}

The present study assessed the effect of a 12-week physical and cognitive training using a $2 \times 2$ factorial design where each intervention (AR and DT) had its own active control condition (ST and CL), leading to four training combinations. In terms of physical performance, all groups showed equivalent improvements in measures of functional capacity, that is, the 6-MWT and the PPT. However, only the AR condition has led to significant improvements in lower body strength, estimated with the chair stand test, independently of cognitive training. As for the cognitive outcomes, all groups showed an improved speed of processing (TMT-A) and inhibition abilities (CWIT-Inhibition), but only participants of the DT training groups, independently of physical training, showed increased task-switching abilities (CWIT-switching). In addition, the level of functional mobility after intervention was significantly associated with task-switching abilities.

Overall, AR training and ST seem to have promoted changes in functional mobility. Both training regimens exerted a beneficial effect on mobility measures like the 6-MWT, as well as on the modified PPT. The ST condition was developed as an active control condition for the AR training. However, we have probably underestimated the potential gains of stretching exercises on our primary outcome measures in a sample of sedentary older adults. Other studies have shown that stretching training can augment gait speed and agility in older women ${ }^{74}$ and can also increase the range of motion, leading to better functional mobility. ${ }^{65}$ 
The equivalent improvements over time in all groups in the 6-MWT and the PPT could be explained by the positive effects of both AR training and ST on functional capacity through different mechanisms. It is also worth pointing out that the movement required to attend training sessions three times a week at the geriatric institute where the study took place may have been sufficient to improve mobility in sedentary participants, as many of them were taking public transportation (subway and bus) to come to their training sessions. Whereas both physical training conditions improved gait speed and physical functional capacities, the AR training condition has led to a greater improvement in lower body strength, as measured with the chair stand test, when compared to the ST group. Thus, the resistance training portion of the AR training condition seems to have led to increased muscle strength, a physical performance outcome that has been associated with cognitive health in older adults. ${ }^{75}$

The beneficial effect of physical exercise alone on executive functioning has been partly reproduced in this study. Previous literature has emphasized the sensitivity of frontally mediated cognitive functions to physical exercise interventions. ${ }^{10,16}$ More specific mechanisms that fall under the executive functions umbrella appear to respond differentially to physical training. In fact, in Barenberg et al's ${ }^{13}$ review, both short- and long-term physical activity interventions yielded improvement in inhibition, whereas only long-term training programs have led to improvement in DT coordination and shifting. Along the same line, Predovan et $\mathrm{al}^{76}$ suggest that switching abilities can be improved after 12 weeks of aerobic training, three times a week. Yet, in the present study, all groups showed improvements in inhibition after intervention, and AR training alone did not lead to improvements in task switching. While these results do not fully support the existing claim that executive functioning can be improved by physical training, the fact that the training regimen of stretching exercises has led to improvements in overall mobility suggests that all participants may have benefited from the physiological changes elicited by physical activity, regardless of the type of training, and that these changes may have promoted better cognitive functioning in terms of processing speed and inhibition. At the same time, our results raise the question about the minimal amount of physical training, regarding intensity, frequency, or total duration, that is necessary to induce changes in cognitive performance, especially in some executive mechanisms, such as task switching. Whereas many studies have implemented physical training programs three times a week for at least 12 weeks, our physical training intervention was reduced to two times a week for 12 weeks to allow for the addition of a cognitive intervention, hence it is possible that this resulted in an insufficient frequency of exercise. Furthermore, it is possible that the moderate intensity of the exercise was not optimal, as some studies suggest that high intensity exercise is more likely to produce cognitive enhancement. ${ }^{77-79}$ Nevertheless, the level of mobility after intervention, measured with the 6-MWT, was strongly associated with task-switching abilities, therefore suggesting a relationship between improved physical functioning and cognitive performance after intervention.

One of the interesting results stemming from this study is the presence of transfer effects on executive functioning after DT training. Dual tasking entails coordination of two tasks that have to be performed concurrently. Many studies have demonstrated that this specific ability to coordinate two tasks can be improved after training, ${ }^{32,53,56,80}$ but the extent of the transfer effect has mostly been observed with similar untrained tasks that involve different stimuli, often referred to as near transfer. Evidences for far transfer, referring to the training-induced improvement in untrained tasks with fairly different features, exist in the literature ${ }^{30,81}$ but still remain scarce. In the present study, participants who took part to the DT training reduced significantly their completion time in the switching condition of the clinical Stroop task (CWIT). This condition presents color words written with incongruent ink colors (eg, the word "blue" printed in red ink) and requires shifting attention throughout the task from the color of the ink (color naming) to the semantic meaning of the word (reading) according to a visual cue. The task-switching abilities involved in this condition are different from the DT processing, as it does not require to perform two tasks simultaneously. However, DT processing and task switching require attentional control and share a certain amount of variance. ${ }^{43}$ As these tasks heavily rely on the integrity of the frontal cortex, it could be argued that enhanced DT processing may have led to brain plasticity mechanisms or adaptations that also favor task-switching capacities. Such transfer effects from one executive task to another after DT training had previously been observed. ${ }^{82}$

Results of the present study did not yield significant additional benefits from combining physical training and cognitive training. These results seem at odd with previous studies using combined interventions. In fact, Fabre et al, ${ }^{37}$ with only eight participants per group, have shown larger improvements in memory performance after 2 months of combined aerobic and cognitive training when compared to single condition. Oswald et $\mathrm{al}^{38}$ have further demonstrated a 
sustained positive effect of combined training on cognitive performance 5 years after the termination of their trial when compared to no treatment control group. However, it is worth noting that participants in this latter study were not randomly assigned to experimental conditions and that no measures of lifestyle habits were taken during the follow-up period, thereby suggesting that individuals involved in the combined condition were perhaps already physically and cognitively active as well as free of medical concerns and were thus more likely to maintain this active lifestyle in the long term. In-line with our results, Linde and Alfermann ${ }^{40}$ and Shatil ${ }^{39}$ did not show additional benefits on cognitive outcome measures of adding a physical training component to a cognitive training. Comparisons to a no treatment control group in studies conducted so far hold alternative explanations for the beneficial effects of training, because only the intervention groups received significant amounts of interpersonal contact with other participants as well as with experimenters. Moreover, participants from combined intervention groups received up to twice as much intervention time than each training group alone. This is not a trivial issue since social engagement has been identified as a valid buffer against cognitive decline s3,84 $^{83}$ and dementia ${ }^{85,86}$ and may thus explain at least in part the cognitive enhancement effect observed in these studies. ${ }^{87}$ In the present study, each intervention group received the same amount of social interactions, the only difference being the type of training program. Results from the present study are partly in-line with another randomized controlled trial involving 126 older adults with subjective cognitive impairment. The authors used a similar factorial design with active control groups and observed no significant beneficial effects of combining physical exercise with cognitive training. ${ }^{88}$ They further excluded the hypothesis of practice effects by comparing cognitive improvements observed in their study with an additional no treatment control group that showed significantly less cognitive improvement than the intervention groups. However, in the present study, the absence of a no treatment control group precludes definitive conclusions about a potential test-retest effect to explain the results. Nevertheless, the selective ameliorations in certain physical and cognitive domains observed only in participants who completed the DT training program or the AR training program strongly suggest a training-induced selective improvement in these outcome measures rather than a general practice effect.

Limitations of this study include the lack of a no treatment control group that could have excluded the potential testretest effects in explaining changes in physical and cognitive functioning. Furthermore, it is difficult to assess the specific effect of the AR training condition on cardiorespiratory fitness due to the lack of an objective measure, such as $\mathrm{VO}_{2}$ max. The objective assessment of cardiorespiratory fitness could have help clarify the contribution of increased cardiorespiratory fitness in the improvement of functional mobility. The use of neuropsychological tests for assessing cognitive functioning in our cognitively healthy sample may also have prevented the finding of more subtle cognitive changes that experimental tasks could have more easily captured. Also, the small number of men included in the study, as well as their unequal distribution among groups due to randomization, precludes analyses of sex differences in the effects of the interventions. Finally, the absence of synergetic effect between physical and cognitive function leaves unanswered the fundamental question about possible interactions between both types of interventions. It is possible that this synergetic effect does not exist, or else that different parameters of exercise and cognitive stimulation could have led to an interaction. The addition of a follow-up assessment many months after the interventions could have revealed stronger gains in the combined intervention, as suggested by Rahe et a ${ }^{58}$ who showed significant benefits of combined training on attention only at the 1-year follow-up assessment.

An interesting avenue in the study of synergetic effect would be to look at the effect of simultaneous (instead of sequential) cognitive and physical activities on cognitive performance. Simultaneous training involves practicing a physical task and a cognitive task at the same time in order to create a motor-cognitive DT or complex training. Increased involvement of cognitive resources for performing motor tasks in older adults has become a well-known phenomenon, ${ }^{52}$ and it justifies the use of more ecological DTs that involve both a physical component and a cognitive component. Motor-cognitive DT training is assumed to stimulate brain activity in areas responsible for performing both tasks at once, which would lead to training-induced improvements in the learned DT, ${ }^{89}$ that may also transfer to similar untrained task. Some studies already attempted this type of combined training and reported promising results. ${ }^{90,91}$ However, the demonstration of superior benefits of simultaneous training compared to sequential training still remains to be done. ${ }^{92}$ A trial comparing directly sequential and simultaneous training is in preparation ${ }^{89}$ and would help clarify the relative benefits as well as the extent of transfer effects in each type of combined training.

Future studies are also needed to examine the optimal duration, frequency, and intensity of physical and cognitive 
training that are required to boost cognitive functioning in older adults. A better understanding of this dose-response relationship could also help implementing optimal conditions to observe potential synergetic effects of physical and cognitive training. Furthermore, inclusion of neuroimaging as well as blood analyses would be a crucial way to find biomarkers that can elucidate the relationship between physical and cognitive training effects on the aging brain.

\section{Conclusion}

This study examined the effects of a 12-week combined physical and cognitive intervention on fitness and neuropsychological outcomes in community-dwelling older adults. The hypothesis that the combined intervention would lead to better executive functioning than either intervention alone was not verified. However, main effects of time were observed on functional capacity measures as well as on speed of processing and inhibition, suggesting that physical and cognitive abilities that are crucial for independent living in the elderly can benefit from various types of intervention. These findings further support the notion that different preventive approaches may be useful to counteract some of the age-related cognitive decline. In addition, this study reported interesting transfer effects of DT training on task-switching abilities. Further studies are still necessary to disentangle the relative contribution of each type of training on specific cognitive functions, to clarify the dose-response relationship between physical training and cognitive enhancement, to directly compare sequential vs simultaneous combined training, and to identify underlying physiological mechanisms that are responsible for the observed effects.

\section{Acknowledgments}

This study was supported by a Canadian Institutes of Health Research (CIHR) grant (\#187596). LD-C was supported by a doctoral fellowship from the CIHR, and LB was supported by the Canada Research Chair Program. The authors would like to thank Dre Kristell Pothier for helpful comments on the article.

\section{Disclosure}

The authors report no conflicts of interest in this work.

\section{References}

1. Hertzog C, Kramer AF, Wilson RS, Lindenberger U. Erichment effects on adult cognitive development: can the functional capacity of older adults be preserved and enhanced? Psychol Sci Public Interest. 2009; $9(1): 1-65$.
2. Ballesteros S, Kraft E, Santana S, Tziraki C. Maintaining older brain functionality: a targeted review. Neurosci Biobehav Rev. 2015;55:453-477.

3. Bherer L. Cognitive plasticity in older adults: effects of cognitive training and physical exercise. Ann N Y Acad Sci. 2015;1337:1-6.

4. Etnier JL, Nowell PM, Landers DM, Sibley BA. A meta-regression to examine the relationship between aerobic fitness and cognitive performance. Brain Res Rev. 2006;52(1):119-130.

5. Dupuy O, Gauthier CJ, Fraser SA, et al. Higher levels of cardiovascular fitness are associated with better executive function and prefrontal oxygenation in younger and older women. Front Hum Neurosci. 2015;9:66

6. Voelcker-Rehage C, Godde B, Staudinger UM. Physical and motor fitness are both related to cognition in old age. Eur J Neurosci. 2010; 31(1):167-176.

7. Barnes DE, Yaffe K, Satariano WA, Tager IB. A longitudinal study of cardiorespiratory fitness and cognitive function in healthy older adults. $J$ Am Geriatr Soc. 2003;51(4):459-465.

8. Sofi F, Valecchi D, Bacci D, et al. Physical activity and risk of cognitive decline: a meta-analysis of prospective studies. J Intern Med. 2011; 269(1):107-117.

9. Smith PJ, Blumenthal JA, Hoffman BM, et al. Aerobic exercise and neurocognitive performance: a meta-analytic review of randomized controlled trials. Psychosom Med. 2010;72(3):239-252.

10. Colcombe $\mathrm{S}, \mathrm{Kramer}$ AF. Fitness effects on the cognitive function of older adults: a meta-analytic study. Psychol Sci. 2003;14(2):125-130.

11. Kramer AF, Hahn S, Cohen NJ, et al. Ageing, fitness and neurocognitive function. Nature. 1999;400(6743):418-419.

12. Hall CD, Smith AL, Keele SW. The impact of aerobic activity on cognitive function in older adults: a new synthesis based on the concept of executive control. Eur J Cognit Psychol. 2001;13:279-300.

13. Barenberg J, Berse T, Dutke S. Executive functions in learning processes: do they benefit from physical activity? Educ Res Rev. 2011;6: 208-222.

14. Colcombe SJ, Kramer AF, Erickson KI, et al. Cardiovascular fitness, cortical plasticity, and aging. Proc Natl Acad Sci U S A. 2004;101(9): 3316-3321.

15. Colcombe SJ, Erickson KI, Scalf PE, et al. Aerobic exercise training increases brain volume in aging humans. J Gerontol A Biol Sci Med Sci. 2006;61(11):1166-1170.

16. Kramer AF, Colcombe S, Erickson K, et al. Effects of aerobic fitness training on human cortical function: a proposal. J Mol Neurosci. 2002; 19(1-2):227-231.

17. Liu-Ambrose T, Nagamatsu LS, Voss MW, Khan KM, Handy TC. Resistance training and functional plasticity of the aging brain: a 12-month randomized controlled trial. Neurobiol Aging. 2012;33(8):1690-1698.

18. Berryman N, Bherer L, Nadeau S, et al. Multiple roads lead to Rome: combined high-intensity aerobic and strength training vs gross motor activities leads to equivalent improvement in executive functions in a cohort of healthy older adults. Age (Dordr). 2014;36(5):9710.

19. Chang YK, Pan CY, Chen FT, Tsai CL, Huang CC. Effect of resistanceexercise training on cognitive function in healthy older adults: a review. J Aging Phys Act. 2012;20(4):497-517.

20. Lustig C, Shah P, Seidler R, Reuter-Lorenz PA. Aging, training, and the brain: a review and future directions. Neuropsychol Rev. 2009; 19(4):504-522.

21. Belleville $\mathrm{S}$, Bherer L. Biomarkers of cognitive training effects in aging. Curr Transl Geriatr Exp Gerontol Rep. 2012;1(2):104-110.

22. Mowszowski L, Batchelor J, Naismith SL. Early intervention for cognitive decline: can cognitive training be used as a selective prevention technique? Int Psychogeriatr. 2010;22(4):537-548.

23. Lussier M, Brouillard P, Bherer L. Limited benefits of heterogeneous dual-task training on transfer effects in older adults. $J$ Gerontol B Psychol Sci Soc Sci. Epub 2015 Nov 23.

24. Lee H, Boot WR, Basak C, et al. Performance gains from directed training do not transfer to untrained tasks. Acta Psychol (Amst). 2012; 139(1):146-158. 
25. Verhaeghen P, Marcoen A, Goossens L. Improving memory performance in the aged through mnemonic training: a meta-analytic study. Psychol Aging. 1992;7(2):242-251.

26. Rebok GW, Carlson MC, Langbaum JB. Training and maintaining memory abilities in healthy older adults: traditional and novel approaches. J Gerontol B Psychol Sci Soc Sci. 2007;62(Spec No 1):53-61.

27. Boron JB, Turiano NA, Willis SL, Schaie KW. Effects of cognitive training on change in accuracy in inductive reasoning ability. J Gerontol B Psychol Sci Soc Sci. 2007;62(3):179-186.

28. Brehmer Y, Westerberg H, Backman L. Working-memory training in younger and older adults: training gains, transfer, and maintenance. Front Hum Neurosci. 2012;6:63.

29. Li SC, Schmiedek F, Huxhold O, Rocke C, Smith J, Lindenberger U. Working memory plasticity in old age: practice gain, transfer, and maintenance. Psychol Aging. 2008;23(4):731-742.

30. Roenker DL, Cissell GM, Ball KK, Wadley VG, Edwards JD. Speedof-processing and driving simulator training result in improved driving performance. Hum Factors. 2003;45(2):218-233.

31. Edwards JD, Wadley VG, Vance DE, Wood K, Roenker DL, Ball KK. The impact of speed of processing training on cognitive and everyday performance. Aging Ment Health. 2005;9(3):262-271.

32. Bherer L, Kramer AF, Peterson MS, Colcombe S, Erickson K, Becic E. Training effects on dual-task performance: are there age-related differences in plasticity of attentional control? Psychol Aging. 2005; 20(4):695-709.

33. Kramer AF, Larish JF, Strayer DL. Training for attentional control in dual task settings: a comparison of young and old adults. J Exp Psychol Appl. 1995;1:50-76.

34. Coelho FG, Andrade LP, Pedroso RV, et al. Multimodal exercise intervention improves frontal cognitive functions and gait in Alzheimer's disease: a controlled trial. Geriatr Gerontol Int. 2013;13(1):198-203.

35. Schwenk M, Zieschang T, Oster P, Hauer K. Dual-task performances can be improved in patients with dementia: a randomized controlled trial. Neurology. 2010;74(24):1961-1968.

36. Suzuki T, Shimada H, Makizako H, et al. Effects of multicomponent exercise on cognitive function in older adults with amnestic mild cognitive impairment: a randomized controlled trial. BMC Neurol. 2012; 12:128.

37. Fabre C, Chamari K, Mucci P, Masse-Biron J, Prefaut C. Improvement of cognitive function by mental and/or individualized aerobic training in healthy elderly subjects. Int J Sports Med. 2002;23(6):415-421.

38. Oswald WD, Gunzelmann T, Rupprecht R, Hagen B. Differential effects of single versus combined cognitive and physical training with older adults: the SimA study in a 5-year perspective. Eur J Ageing. 2006;3(4): 179-192.

39. Shatil E. Does combined cognitive training and physical activity training enhance cognitive abilities more than either alone? A four-condition randomized controlled trial among healthy older adults. Front Aging Neurosci. 2013;5:8.

40. Linde K, Alfermann D. Single versus combined cognitive and physical activity effects on fluid cognitive abilities of healthy older adults: a 4-month randomized controlled trial with follow-up. J Aging Phys Act. 2014;22(3):302-313.

41. Jaeggi SM, Buschkuehl M, Jonides J, Perrig WJ. Improving fluid intelligence with training on working memory. Proc Natl Acad Sci U S A. 2008;105(19):6829-6833.

42. Karbach J, Mang S, Kray J. Transfer of task-switching training in older age: the role of verbal processes. Psychol Aging. 2010;25(3): 677-683.

43. Miyake A, Friedman NP, Emerson MJ, Witzki AH, Howerter A. The unity and diversity of executive functions and their contributions to complex "frontal lobe" tasks: a latent variable analysis. Cogn Psychol. 2000;41:49-100.

44. Cahn-Weiner DA, Malloy PF, Boyle PA, Marran M, Salloway S. Prediction of functional status from neuropsychological tests in communitydwelling elderly individuals. Clin Neuropsychol. 2000;14(2): 187-195.
45. Carlson MC, Fried LP, Xue QL, Bandeen-Roche K, Zeger SL, Brandt J. Association between executive attention and physical functional performance in community-dwelling older women. J Gerontol B Psychol Sci Soc Sci. 1999;54(5):S262-S270.

46. Royall DR, Palmer R, Chiodo LK, Polk MJ. Declining executive control in normal aging predicts change in functional status: the Freedom House Study. J Am Geriatr Soc. 2004;52(3):346-352.

47. Johnson JK, Lui LY, Yaffe K. Executive function, more than global cognition, predicts functional decline and mortality in elderly women. J Gerontol A Biol Sci Med Sci. 2007;62(10):1134-1141.

48. Bell-McGinty S, Podell K, Franzen M, Baird AD, Williams MJ. Standard measures of executive function in predicting instrumental activities of daily living in older adults. Int J Geriatr Psychiatry. 2002; 17(9):828-834.

49. Verhaeghen P, Cerella J. Aging, executive control, and attention: a review of meta-analyses. Neurosci Biobehav Rev. 2002;26(7):849-857.

50. Holtzer R, Stern Y, Rakitin BC. Predicting age-related dual-task effects with individual differences on neuropsychological tests. Neuropsychology. 2005;19(1):18-27.

51. Springer S, Giladi N, Peretz C, Yogev G, Simon ES, Hausdorff JM. Dual-tasking effects on gait variability: the role of aging, falls, and executive function. Mov Disord. 2006;21(7):950-957.

52. Woollacott M, Shumway-Cook A. Attention and the control of posture and gait: a review of an emerging area of research. Gait Posture. 2002; 16(1):1-14.

53. Bherer L, Kramer AF, Peterson MS, Colcombe S, Erickson K, Becic E. Testing the limits of cognitive plasticity in older adults: application to attentional control. Acta Psychol (Amst). 2006;123(3):261-278.

54. Bherer L, Kramer AF, Peterson MS, Colcombe S, Erickson K, Becic E. Transfer effects in task-set cost and dual-task cost after dual-task training in older and younger adults: further evidence for cognitive plasticity in attentional control in late adulthood. Exp Aging Res. 2008;34(3): 188-219.

55. Liepelt R, Strobach T, Frensch P, Schubert T. Improved intertask coordination after extensive dual-task practice. QJ Exp Psychol (Hove). 2011; 64(7):1251-1272.

56. Lussier M, Gagnon C, Bherer L. An investigation of response and stimulus modality transfer effects after dual-task training in younger and older. Front Hum Neurosci. 2012;6:129.

57. Safer DL, Hugo EM. Designing a control for a behavioral group therapy. Behav Ther. 2006;37(2):120-130.

58. Rahe J, Petrelli A, Kaesberg S, Fink GR, Kessler J, Kalbe E. Effects of cognitive training with additional physical activity compared to pure cognitive training in healthy older adults. Clin Interv Aging. 2015;10: 297-310.

59. Bherer L, Erickson KI, Liu-Ambrose T. A review of the effects of physical activity and exercise on cognitive and brain functions in older adults. J Aging Res. 2013;2013:657508.

60. Yesavage JA. Geriatric Depression Scale. Psychopharmacol Bull. 1988; 24(4):709-711

61. Desjardins-Crepeau L, Berryman N, Vu TT, et al. Physical functioning is associated with processing speed and executive functions in communitydwelling older adults. J Gerontol B Psychol Sci Soc Sci. 2014;69(6): 837-844.

62. Folstein MF, Folstein SE, McHugh PR. "Mini-mental state". A practical method for grading the cognitive state of patients for the clinician. J Psychiatr Res. 1975;12(3):189-198.

63. Wechsler D. Wechsler Adult Intelligence Scale - Third Edition: Administration and Scoring Manual. San Antonio, TX: The Psychological Corporation; 1997.

64. Borg G. Borg's Perceived Exertion and Pain Scales. Champaign, IL: Human Kinetics; 1998.

65. Stanziano DC, Roos BA, Perry AC, Lai S, Signorile JF. The effects of an active-assisted stretching program on functional performance in elderly persons: a pilot study. Clin Interv Aging. 2009;4:115-120.

66. Lezak M, Howison DB, Bigler ED, Tranel D. Neuropsychological Assessment. 5th ed. New York, NY: Oxford University Press; 2012. 
67. Delis DC, Kaplan E, Kramer JH. The Delis-Kaplan Executive Function System: Technical Manual. San Antonio, TX: The Psychological Corporation; 2001.

68. Stroop JR. Studies of interference in serial verbal reactions. $J \operatorname{Exp}$ Psychol. 1935;18:643-661.

69. Reitan RM. Validity of the trail making test as an indicator of organic brain damage. Percept Mot Skills. 1958;8:271-276.

70. Della Sala S, Baddeley A, Papagno C, Spinnler H. Dual-task paradigm: a means to examine the central executive. Ann N Y Acad Sci. 1995; 769:161-171.

71. Bautmans I, Lambert M, Mets T. The six-minute walk test in community dwelling elderly: influence of health status. BMC Geriatr. 2004;4:6.

72. Reuben DB, Siu AL. An objective measure of physical function of elderly outpatients. The Physical Performance Test. J Am Geriatr Soc. 1990;38(10):1105-1112.

73. Podsiadlo D, Richardson S. The timed "Up \& Go": a test of basic functional mobility for frail elderly persons. J Am Geriatr Soc. 1991; 39(2):142-148.

74. Gajdosik RL, Vander Linden DW, McNair PJ, Williams AK, Riggin TJ. Effects of an eight-week stretching program on the passive-elastic properties and function of the calf muscles of older women. Clin Biomech (Bristol, Avon). 2005;20(9):973-983.

75. Boyle PA, Buchman AS, Wilson RS, Leurgans SE, Bennett DA. Association of muscle strength with the risk of Alzheimer disease and the rate of cognitive decline in community-dwelling older persons. Arch Neurol. 2009;66(11):1339-1344.

76. Predovan D, Fraser SA, Renaud M, Bherer L. The effect of three months of aerobic training on stroop performance in older adults. J Aging Res. 2012;2012:269815.

77. Angevaren M, Vanhees L, Wendel-Vos W, et al. Intensity, but not duration, of physical activities is related to cognitive function. Eur $J$ Cardiovasc Prev Rehabil. 2007;14(6):825-830.

78. Brown BM, Peiffer JJ, Sohrabi HR, et al; AIBL Research Group. Intense physical activity is associated with cognitive performance in the elderly. Transl Psychiatry. 2012;2:e191.

79. Tierney MC, Moineddin R, Morra A, Manson J, Blake J. Intensity of recreational physical activity throughout life and later life cognitive functioning in women. J Alzheimers Dis. 2010;22(4):1331-1338.

80. Erickson KI, Colcombe SJ, Wadhwa R, et al. Training-induced plasticity in older adults: effects of training on hemispheric asymmetry. Neurobiol Aging. 2007;28(2):272-283
81. Cassavaugh ND, Kramer AF. Transfer of computer-based training to simulated driving in older adults. Appl Ergon. 2009;40(5):943-952.

82. Bier B, de Boysson C, Belleville S. Identifying training modalities to improve multitasking in older adults. Age (Dordr). 2014;36(4):9688.

83. Barnes LL, Mendes de Leon CF, Wilson RS, Bienias JL, Evans DA. Social resources and cognitive decline in a population of older African Americans and whites. Neurology. 2004;63(12):2322-2326.

84. Lovden M, Ghisletta P, Lindenberger U. Social participation attenuates decline in perceptual speed in old and very old age. Psychol Aging. 2005; 20(3):423-434.

85. Fabrigoule C, Letenneur L, Dartigues JF, Zarrouk M, Commenges D, Barberger-Gateau P. Social and leisure activities and risk of dementia: a prospective longitudinal study. J Am Geriatr Soc. 1995;43(5): 485-490.

86. Wang HX, Karp A, Winblad B, Fratiglioni L. Late-life engagement in social and leisure activities is associated with a decreased risk of dementia: a longitudinal study from the Kungsholmen project. Am J Epidemiol. 2002;155(12):1081-1087.

87. Law LL, Barnett F, Yau MK, Gray MA. Effects of combined cognitive and exercise interventions on cognition in older adults with and without cognitive impairment: a systematic review. Ageing Res Rev. 2014;15: $61-75$.

88. Barnes DE, Santos-Modesitt W, Poelke G, et al. The Mental Activity and eXercise (MAX) trial: a randomized controlled trial to enhance cognitive function in older adults. JAMA Intern Med. 2013;173(9):797-804.

89. Schaefer S, Schumacher V. The interplay between cognitive and motor functioning in healthy older adults: findings from dual-task studies and suggestions for intervention. Gerontology. 2011;57(3):239-246.

90. Eggenberger P, Schumacher V, Angst M, Theill N, de Bruin ED. Does multicomponent physical exercise with simultaneous cognitive training boost cognitive performance in older adults? A 6-month randomized controlled trial with a 1-year follow-up. Clin Interv Aging. 2015;10: 1335-1349.

91. Theill N, Schumacher V, Adelsberger R, Martin M, Jancke L. Effects of simultaneously performed cognitive and physical training in older adults. BMC Neurosci. 2013;14:103.

92. Kraft E. Cognitive function, physical activity, and aging: possible biological links and implications for multimodal interventions. Neuropsychol Dev Cogn B Aging Neuropsychol Cogn. 2012;19(1-2) 248-263.
Clinical Interventions in Aging

\section{Publish your work in this journal}

Clinical Interventions in Aging is an international, peer-reviewed journal focusing on evidence-based reports on the value or lack thereof of treatments intended to prevent or delay the onset of maladaptive correlates of aging in human beings. This journal is indexed on PubMed Central, MedLine,

\section{Dovepress}

CAS, Scopus and the Elsevier Bibliographic databases. The manuscript management system is completely online and includes a very quick and fair peer-review system, which is all easy to use. Visit http://www.dovepress. com/testimonials.php to read real quotes from published authors. 